\title{
Fabrication of Highly Robust and Conductive Ion Gels Based on the Combined Strategies of Double-Network, Composite, and High- Functionality Cross-Linkers
}

Kiran Shahzadi, ${ }^{\dagger}+$ Wenjuan Xiong, ${ }^{\dagger *}$ Mehdihasan Shekh, ${ }^{\dagger}, \star$ Florian J. Stadler, ${ }^{\dagger}$ Zhi-Chao Yan,,$^{\dagger} *$

${ }^{\dagger}$ Shenzhen Key Laboratory of Polymer Science and Technology, Guangdong Research Center for Interfacial Engineering of Functional Materials, College of Materials Science and Engineering, Shenzhen University, Shenzhen 518055, China

$\ddagger$ Key Laboratory of Optoelectronic Devices and Systems of Ministry of Education and Guangdong Province, College of Optoelectronic Engineering, Shenzhen University, Shenzhen 518060, China

*Corresponding author, email: yanzhch@ szu.edu.cn 


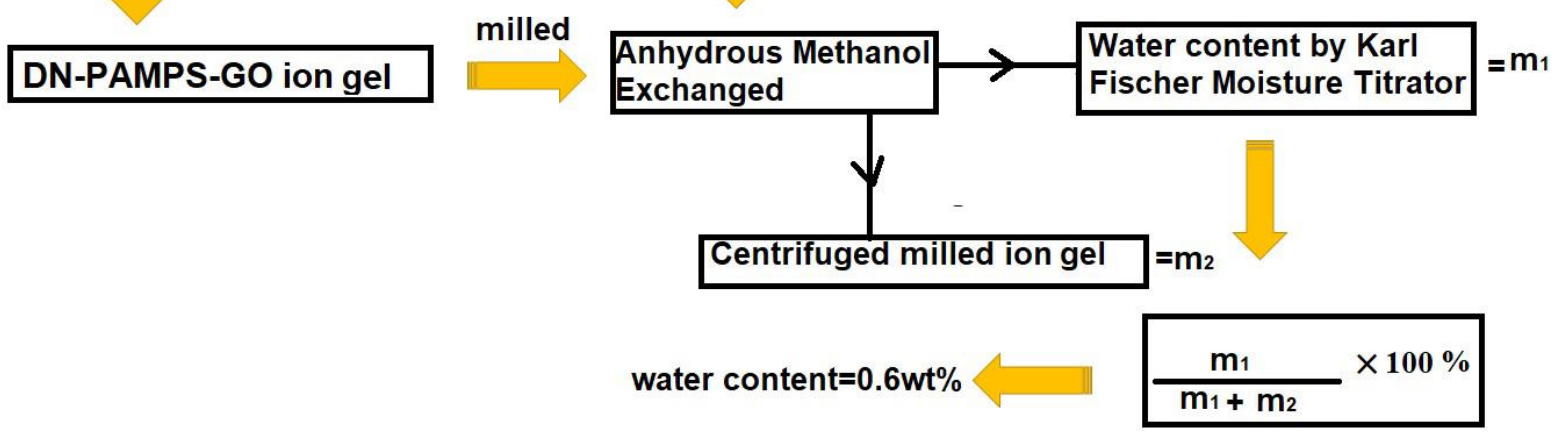

Figure S1. Scheme to determine the water content of the DN-PAMPS-GO ion gel

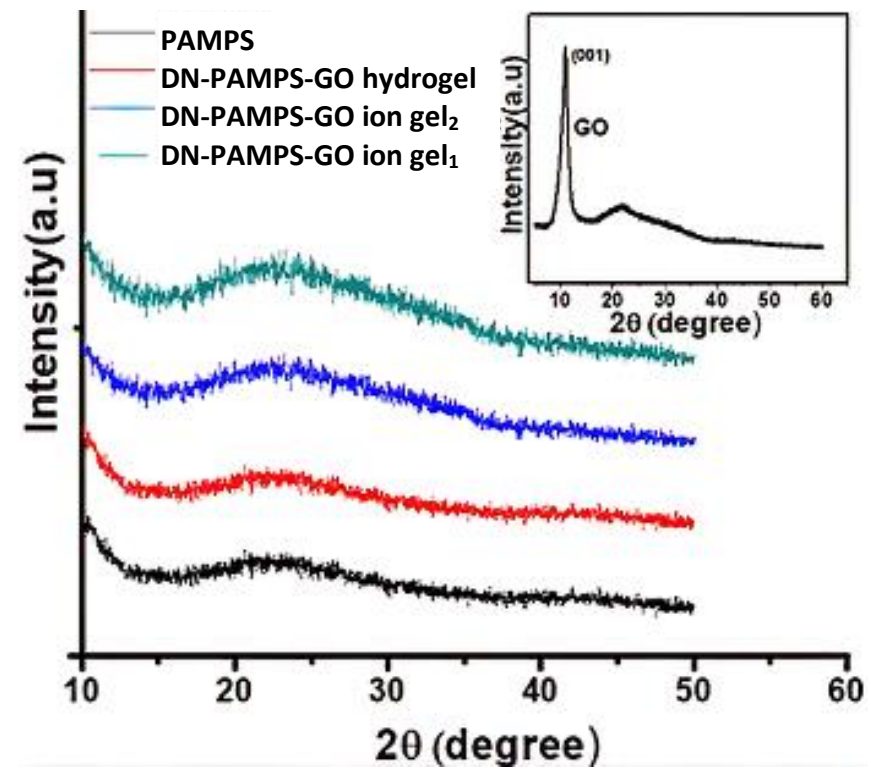

Figure S2. XRD spectra for PAMPS hydrogel, DN-PAMPS-GO hydrogel, DN-PAMPS-GO ion gel $_{2}$, and DN-PAMPS-GO ion gel (from bottom to top). The inset shows the XRD peak for GO. 

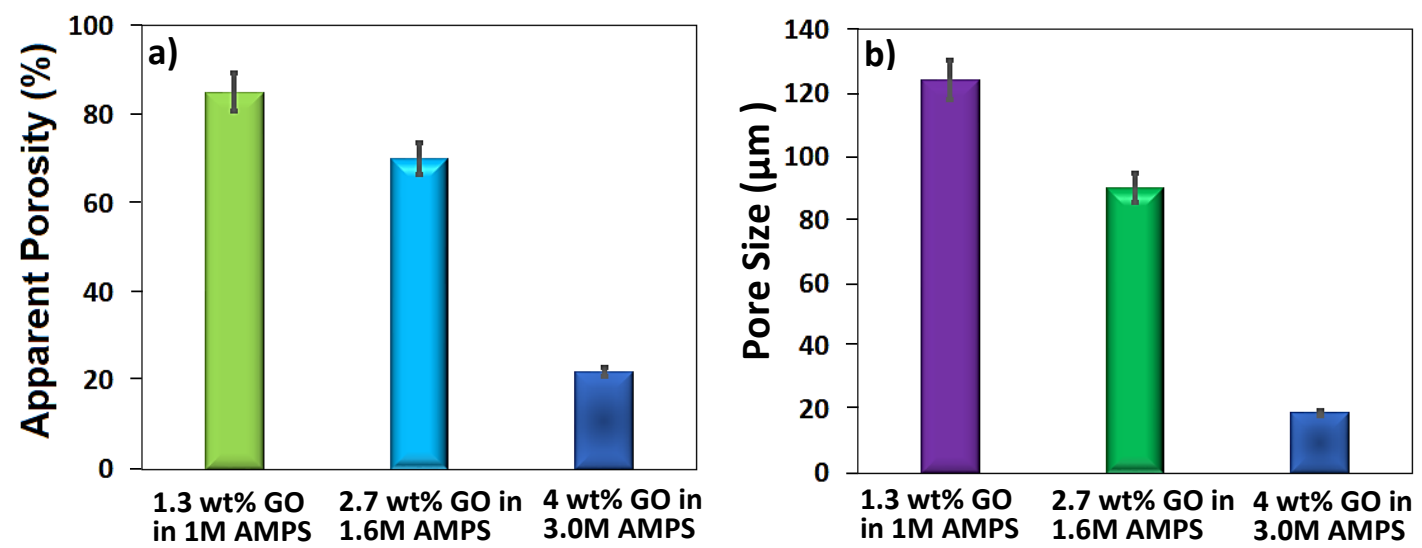

Figure S3. (a) Apparent porosity and (b) pore size for different modified DN-PAMPS-GO hydrogels, determined from SEM images.

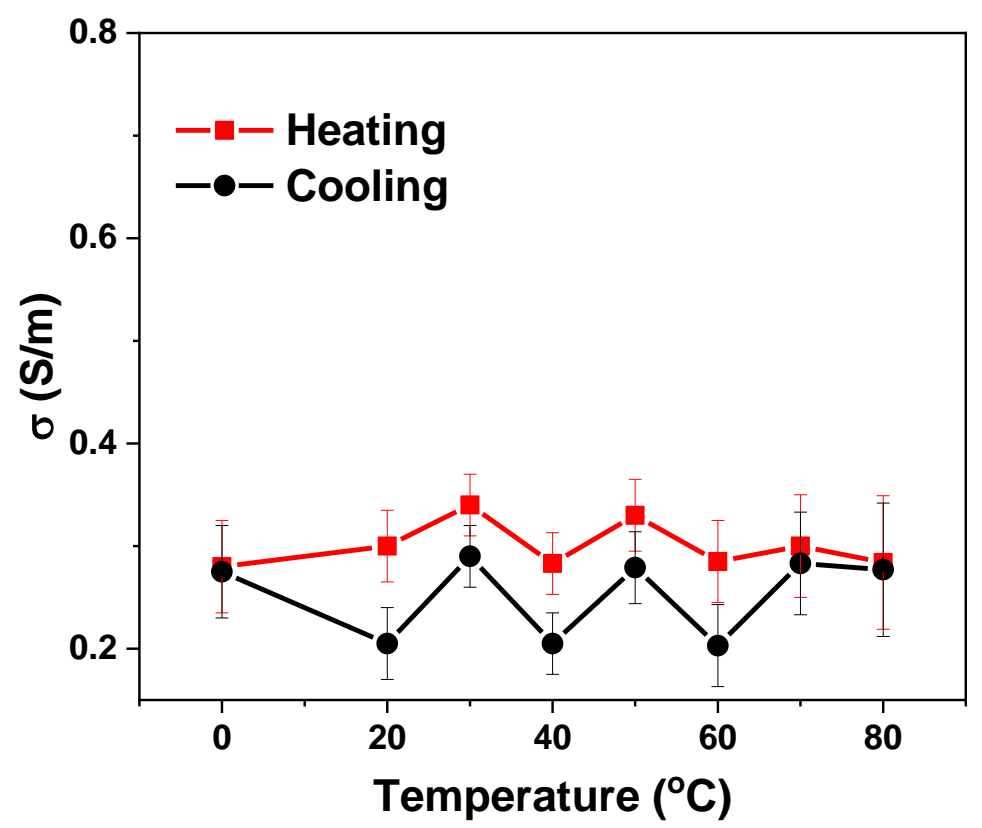

Figure S4. Conductivity of DN-PAMPS-GO ion gel $_{2}$ at different temperatures 

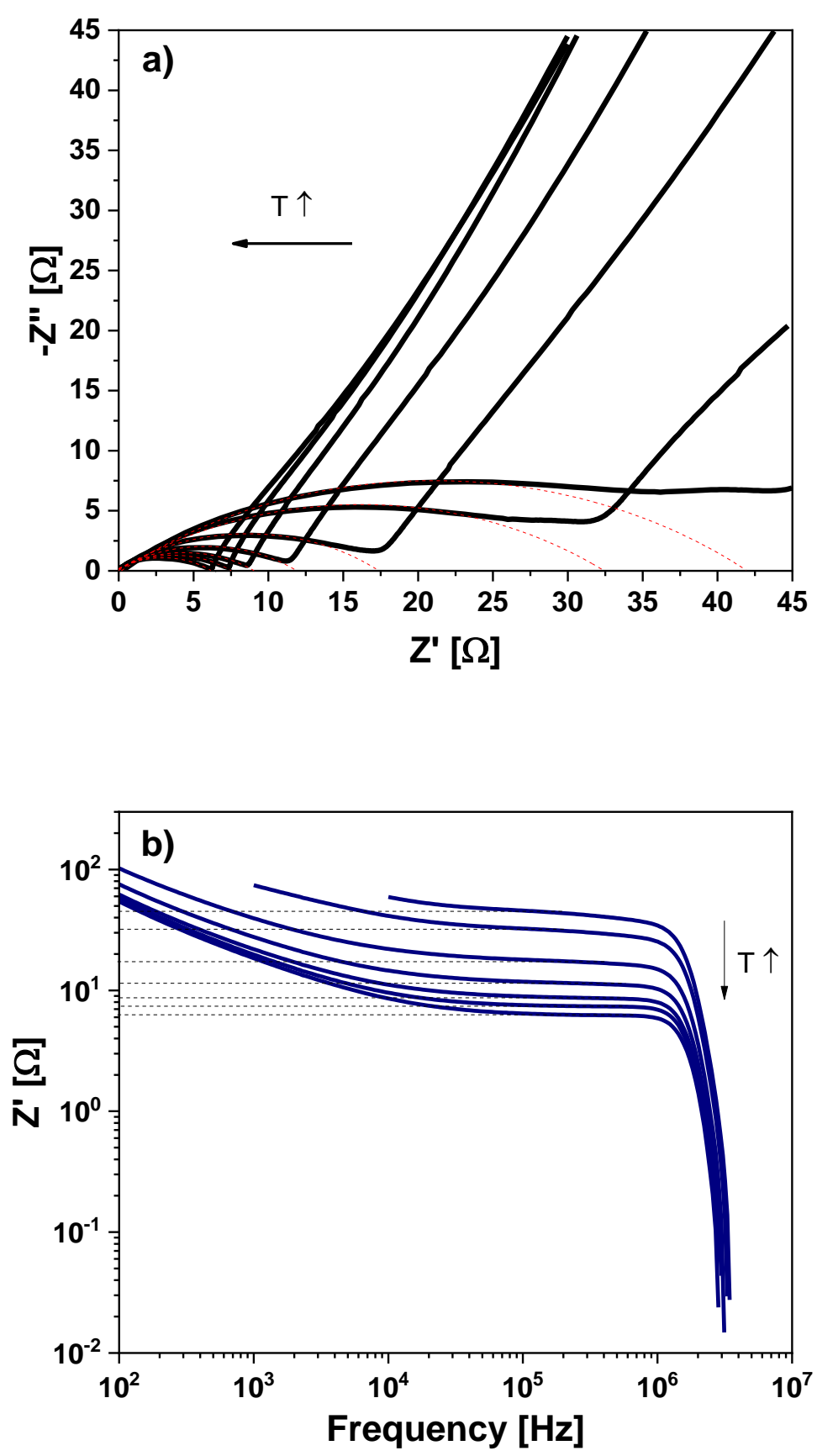

Figure S5. (a) The Nyquist plot of the complex impedance for DN-PAMPS-GO ion gel $_{1}$. The dotted curves are fitted based on the formula for a circle, and the resistance is obtained from the $x$-axis intercept. (b) The real component $\left(Z^{\prime}\right)$ of impedance as a function of frequency. The dotted line denotes the resistance. From right to left in (a) and from top to bottom in (b), the temperatures are sequentially $22,30,42,53,62,71$, and $84{ }^{\circ} \mathrm{C}$. 


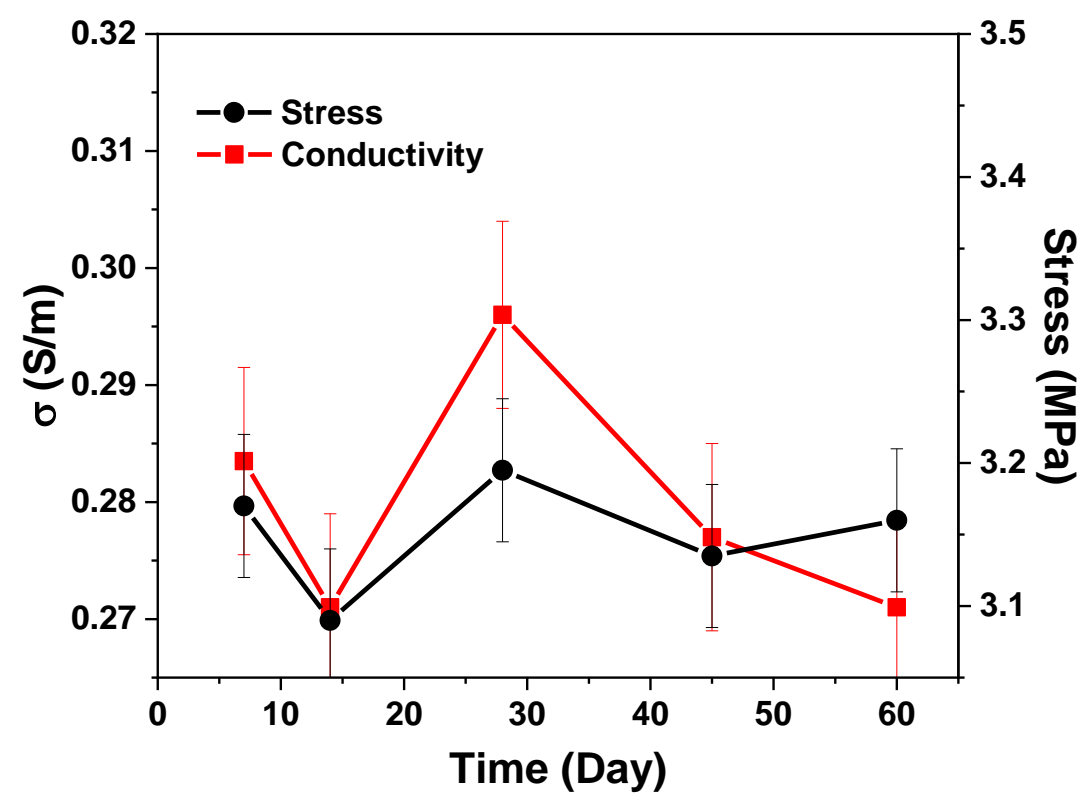

Figure S6. Conductivity and tensile stress stability of DN-PAMPS-GO ion gel 2

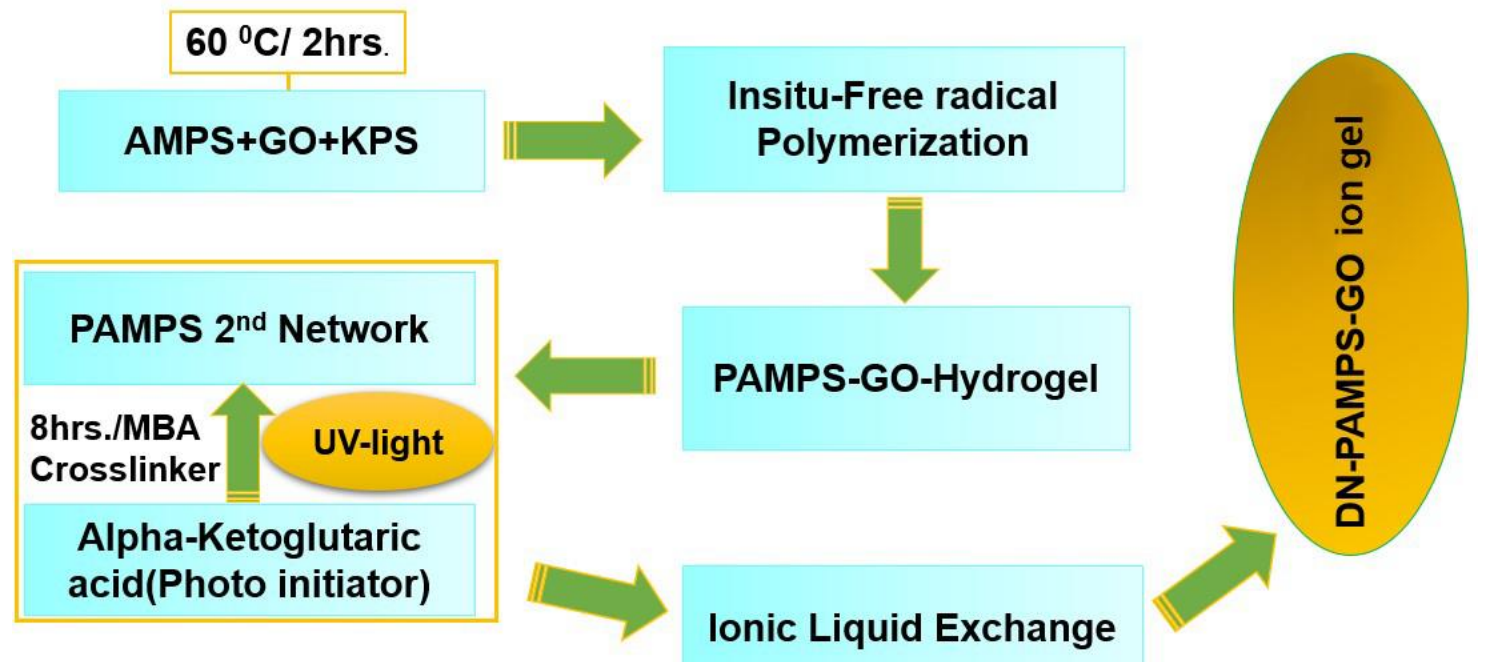

Figure S7. Procedure for fabricating DN-PAMPS-GO ion gels. 